\title{
Molecular analyses of shallow-water zooxanthellate zoanthids (Cnidaria: Hexacorallia) from Taiwan and their Symbiodinium spp
}

\author{
James Davis Reimer ${ }^{*}$ Y Yuka Irei, Takuma Fujii and Sung-Yin Yang
}

\begin{abstract}
Background: Although we previously reported on surveys of shallow-water zoanthid (Anthozoa: Hexacorallia: Zoantharia) species diversity from localities in Taiwan, no study has yet examined the molecular phylogeny of the majority of specimens, nor has any study examined zoanthid zooxanthellae (Symbiodinium spp.) diversity in Taiwan. As many phylogenetic results have been reported from Japan, a critical step in testing DNA identification of zoanthids and their symbionts is the application of these techniques to specimens from neighboring regions such as Taiwan. We utilized three DNA markers (mitochondrial cytochrome oxidase subunit I (COI), mitochondrial 16S ribosomal DNA (mt 165 rDNA), and the nuclear internal transcribed spacer region of ribosomal DNA (ITS-rDNA)) to examine the molecular phylogeny of 110 zooxanthellate specimens collected from 10 localities in Keelung, Kenting, and Green Island. Additionally, we report on the types of Symbiodinium spp. (zooxanthellae) from the three regions above plus the Penghu Islands, utilizing sequences of ITS2-rDNA.

Results: Most specimens in the suborder Brachycnemina belonged to previously reported species. However, it appears that there are still some undescribed Palythoa species groups in the northwestern Pacific that are cryptic in having small colony sizes ( $<5$ polyps) and occurring in low numbers. With regards to Symbiodinium spp. diversity, Palythoa tuberculosa and Palythoa mutuki associated with subclade C1 and related types, Zoanthus sansibaricus associated with subclade C3-derived types, and Zoanthus kuroshio that harbored C15/C91-related Symbiodinium. Notably, $1 / 2$ of the $P$. tuberculosa specimens from the Penghu Islands harbored a previously unreported clade $C$ symbiont, and two Zoanthus. vietnamensis specimens from Keelung also had a unique symbiont type within clade $C$.

Conclusions: It appears that undescribed Palythoa species in the northwestern Pacific may be widely distributed yet low in numbers, often cryptic in their habitat, and with a small colony size. As well, it is clear that much work remains to understand zoanthid-symbiont associations in Taiwan.
\end{abstract}

Keywords: Marine biodiversity; COI; mt 16S rDNA; ITS-rDNA; Taiwan; Zoanthid; Zooxanthellae

\section{Background}

Zoanthids are an order (Zoantharia $=$ Zoanthidea) of Hexacorallia (Cnidaria: Anthozoa) and are common benthos in tropical and subtropical shallow waters (e.g., Karlson 1980). Species of the zooxanthellate genera Zoanthus (family Zoanthidae) and Palythoa (Sphenopidae) within the suborder Brachycnemina are usually dominant components of live reef cover, particularly on coral reef tops and reef edges (Burnett et al. 1997; Swain 2010; Irei

\footnotetext{
* Correspondence: jreimer@sci.u-ryukyu.ac.jp

Molecular Invertebrate Systematics and Ecology Laboratory, Graduate School of Engineering and Science, Univesity of the Ryukyus, Senbaru 1, Nishihara, Okinawa 903-0213, Japan
}

et al. 2011). Nevertheless, species identification in both genera is problematic due to much intraspecific variation and plasticity (Burnett et al. 1994, 1997; Reimer et al. 2004; Ong et al. 2013) and a high "synonymy load" due to inadvertent redescriptions of the same species from different localities (Burnett et al. 1997).

Recent examinations of zoanthids using phylogenetic methods have fostered a new understanding of their species diversity and evolutionary relationships (Burnett et al. 1994, 1997; Reimer et al. 2004; Sinniger et al. 2005, 2013; Swain 2010), and much research has focused on zoanthids in Japanese waters in the northwestern Pacific (e.g., Reimer et al. 2006a,c, 2007c). In Taiwan, recent

\section{슬}


work using morphological field identification resulted in a brachycnemic zoanthid species list for the region (Reimer et al. 2011b), but specimens (excepting Acrozoanthus in Reimer et al. 2010) have not yet been examined using molecular methods.

Many brachycnemic zoanthids are in symbioses with endosymbiotic dinoflagellates of the genus Symbiodinium (= zooxanthellae). Not only zoanthids, but also many scleractinian corals, actiniarians, giant clams, and foraminifers are known to harbor Symbiodinium, and zooxanthellate organisms are therefore responsible for creating much of the architecture of coral reef ecosystems. Based on molecular phylogenetic studies, Symbiodinium is currently divided into nine clades of unknown taxonomic rank (Pochon and Gates 2010), and there are numerous subclades with different associated host species within each clade (e.g., LaJeunesse 2002, 2005; LaJeunesse et al. 2003, 2004), each potentially having different physiologies (Tchernov et al. 2004). For these reasons, research on Symbiodinium diversity is therefore needed to clearly understand the diversity of coral reef and shallow-water ecosystems, and also help anticipate what changes may occur under the threat of global warming.

Recent work on Symbiodinium in brachycnemic zoanthids in Japan revealed different levels of specificity in symbiont-host relationships. Species of the genus Palythoa (family Sphenopidae) generally associate with subclade C1-related Symbiodinium in Japan and the Indian Ocean, and with clade $\mathrm{D}$ in the Indian Ocean (Burnett 2002, Reimer et al. 2006d, Reimer and Todd 2009). However, there appears to be greater variation within the widely distributed species Zoanthus sansibaricus (family Zoanthidae), which is known in the Ryukyu Islands to be associated with subclade A1 in intertidal areas with high light levels, with subclade $\mathrm{C} 1 \mathrm{z}$ sensu Reimer et al. (2007b) in most areas (Reimer et al. 2006e, 2007b), and with another subclade (related to $\mathrm{C} 1$ ) at $>7 \mathrm{~m}$ in depth (Kamezaki et al. 2013).

In this study, we examined (1) zooxanthellate, shallowwater zoanthid species of Taiwanese waters first reported in Reimer et al. (2011b), this time utilizing molecular techniques and discuss the implications of our results and (2) Symbiodinium of the same zoanthid specimens plus of additional new specimens from the Penghu Islands in the Taiwan Strait. Taiwan, while close to Okinawa, has a variety of subtropical and tropical environments that differ from Okinawa, and there is the possibility of differing symbiont associations. For example, the Keelung region of northeastern Taiwan is much cooler in winter than waters in Okinawa, and more reminiscent of environments along the Pacific coast of mainland Japan (Chen 1999; Chen and Shashank 2009), while areas of Green Island off southeastern Taiwan and the coastal area of Kenting (southern Taiwan) have tropical coral reefs. The Penghu Islands, to the west of the main island of Taiwan, are heavily influenced by seasonal monsoons, and waters are often turbid (Huang 2012a, b). Therefore, knowledge of zoanthid-symbiont associations in Taiwan can help expand upon various recent observations of zoanthid-symbiont symbioses from Japan.

\section{Methods}

Surveys of zoanthids in Taiwan were carried out between September 2009 and September 2010 by snorkeling and scuba diving, with additional surveys in December 2011 and September 2012. Twelve investigated sites were divided over four different regions: (1) Kenting, southern Taiwan (Wanlitung, Tiaoshi, Hobihoo, and Longkeng sites; sampling permission \#989094/1400); (2) Green Island (Lyudao in Chinese), off the southeastern coast of Taiwan (Gueiwan, Zolo, Shihlang, Dabaisha, and Matichao sites); (3) Keelung, northeastern Taiwan (Yeliu and Bitouchiao), and (4) the Penghu Islands off the west coast of Taiwan in the Taiwan Strait (Wangan) (Table 1). Some of these surveys and specimens were previously reported in Reimer et al. (2011b). The Keelung region primarily consists of subtropical non-reefal coral communities (Chen 1999; Chen and Shashank 2009), while the other two regions are coral reef ecosystems. In total, 110 specimens were collected (Table 1).

Specimens were photographed in situ prior to collection for characterization of external morphological features (oral disc diameter, color and patterns, and tentacle numbers). Identification of all zooxanthellate species followed Reimer (2010) and Reimer et al. (2011b) except for Acrozoanthus specimens identified following Ryland (1997) and Reimer et al. (2011a). All specimens were identified as in Reimer et al. (2011b) unless otherwise noted (see "Results"). Results for the formally undescribed and azooxanthellate Palythoa sp. "tokashiki" as reported in Reimer et al. (2011b) will be separately reported when this species is formally described.

Specimens were preserved in $70 \%$ or $99.5 \%$ ethanol, and examined at the Univ. of the Ryukyus (UR). Currently, specimens are deposited at the 1st author's laboratory at the UR, but once additional examinations are completed, specimens will be deposited at the National Museum of Natural Science (NMNS), Taichung, Taiwan.

\section{DNA extraction and polymerase chain reaction (PCR) amplification}

DNA was extracted from specimens either with a guanidine method described by Sinniger et al. (2010) or using a spin column DNeasy Blood \& Tissue kit (Qiagen, Tokyo, Japan) following the manufacturer's protocol. The PCR amplification of the extracted genomic DNA was performed using a HotStarTaq Plus Master Mix Kit (Qiagen) following the manufacturer's instructions. Three 
Table 1 Identity of zoanthid specimens from Taiwan examined in this study, their collection information, and associated GenBank accession numbers

\begin{tabular}{|c|c|c|c|c|c|c|c|c|c|c|}
\hline $\begin{array}{l}\text { Specimen } \\
\text { number }\end{array}$ & Location & Region & Date* & $\begin{array}{l}\text { Depth } \\
(\mathrm{m})\end{array}$ & Collector & $\begin{array}{l}\text { mt 16S } \\
\text { rDNA }\end{array}$ & $\mathrm{COI}$ & ITS-rDNA & $\begin{array}{l}\text { Symbiodinium } \\
\text { ITS-rDNA }\end{array}$ & Identity \\
\hline 1582 & Wanli Tung & Kenting & 04-Sep-09 & 4 & JD Reimer & KF499618 & NA & NA & NA & $\begin{array}{l}\text { Palythoa } \\
\text { tuberculosa }\end{array}$ \\
\hline 1583 & Wanli Tung & Kenting & 04-Sep-09 & 4 & JD Reimer & KF499617 & NA & NA & NA & P. tuberculosa \\
\hline 1584 & Wanli Tung & Kenting & 04-Sep-09 & 4 & JD Reimer & KF499616 & NA & NA & NA & P. tuberculosa \\
\hline 1585 & Wanli Tung & Kenting & 04-Sep-09 & 5 & JD Reimer & NA & NA & NA & NA & P. tuberculosa \\
\hline 1586 & Wanli Tung & Kenting & 04-Sep-09 & 5 & JD Reimer & KF499615 & NA & NA & KF499805 & P. tuberculosa \\
\hline 1587 & Wanli Tung & Kenting & 04-Sep-09 & 4 & JD Reimer & KF499666 & KF499698 & NA & NA & P. tuberculosa \\
\hline 1588 & Wanli Tung & Kenting & 04-Sep-09 & 2 & JD Reimer & KF499665 & KF499699 & NA & NA & P. tuberculosa \\
\hline 1589 & Wanli Tung & Kenting & 04-Sep-09 & 1 & JD Reimer & KF499667 & KF499706 & NA & NA & $\begin{array}{l}\text { Zoanthus } \\
\text { vietnamensis }\end{array}$ \\
\hline 1590 & Wanli Tung & Kenting & 04-Sep-09 & 1 & JD Reimer & KF499668 & KF499705 & NA & NA & Z. vietnamensis \\
\hline 1591 & Wanli Tung & Kenting & 04-Sep-09 & 1 & JD Reimer & NA & NA & NA & NA & Z. sansibaricus \\
\hline 1592 & Wanli Tung & Kenting & 04-Sep-09 & 1 & JD Reimer & NA & NA & NA & NA & Z. sansibaricus \\
\hline 1593 & Wanli Tung & Kenting & 04-Sep-09 & 1 & JD Reimer & NA & NA & NA & NA & Z. sansibaricus \\
\hline 1594 & Wanli Tung & Kenting & 04-Sep-09 & 2 & JD Reimer & KF499663 & KF499695 & NA & KF499794 & $\begin{array}{l}\text { Palythoa sp. } \\
\text { "sakurajimensis" }\end{array}$ \\
\hline 1595 & Wanli Tung & Kenting & 04-Sep-09 & 2 & JD Reimer & KF499661 & KF499697 & NA & KF499816 & $\begin{array}{l}\text { Palythoa sp. } \\
\text { "sakurajimensis" }\end{array}$ \\
\hline 1596 & Wanli Tung & Kenting & 04-Sep-09 & 2 & JD Reimer & NA & KF499704 & NA & NA & Palythoa mutuki \\
\hline 1597 & Wanli Tung & Kenting & 04-Sep-09 & 1 & JD Reimer & KF499 & KF499696 & KF499778 & NA & $\begin{array}{l}\text { Palythoa sp. } \\
\text { "sakurajimensis" }\end{array}$ \\
\hline 1598 & Wanli Tung & Kenting & 04-Sep-09 & NA & K Shashank & KF499620 & NA & NA & KF499806 & P. tuberculosa \\
\hline 1599 & Wanli Tung & Kenting & 04-Sep-09 & NA & K Shashank & KF499619 & KF499693 & NA & KF499807 & P. tuberculosa \\
\hline 1600 & Tiao-shi & Kenting & 04-Sep-09 & 8 & JD Reimer & KF499621 & KF499692 & NA & NA & P. tuberculosa \\
\hline 1601 & Tiao-shi & Kenting & 04-Sep-09 & 6 & JD Reimer & KF499622 & KF499691 & NA & NA & P. tuberculosa \\
\hline 1602 & Tiao-shi & Kenting & 04-Sep-09 & 5 & JD Reimer & KF499623 & KF499690 & NA & NA & P. tuberculosa \\
\hline 1603 & Tiao-shi & Kenting & 04-Sep-09 & 5 & JD Reimer & KF499624 & KF499689 & NA & NA & P. tuberculosa \\
\hline 1604 & Tiao-shi & Kenting & 04-Sep-09 & 5 & JD Reimer & KF499625 & KF499688 & NA & NA & P. tuberculosa \\
\hline 1605 & Tiao-shi & Kenting & 04-Sep-09 & 5 & JD Reimer & NA & NA & NA & NA & P. tuberculosa \\
\hline 1606 & Tiao-shi & Kenting & 04-Sep-09 & 5 & JD Reimer & NA & NA & NA & NA & P. tuberculosa \\
\hline 1616 & Ho-bi-hoo & Kenting & 04-Sep-09 & 9 & JD Reimer & KF499626 & KF499687 & NA & NA & P. tuberculosa \\
\hline 1617 & Yeliu & Keelung & 06-Sep-09 & 1 & JD Reimer & KF499627 & KF499745 & KF499767 & KF499787 & P. mutuki \\
\hline 1618 & Yeliu & Keelung & 06-Sep-09 & 2 & JD Reimer & KF499638 & KF499744 & KF499764 & NA & P. mutuki \\
\hline 1619 & Yeliu & Keelung & 06-Sep-09 & 2 & JD Reimer & KF499637 & KF499743 & NA & KF499827 & P. mutuki \\
\hline 1620 & Yeliu & Keelung & 06-Sep-09 & 1 & JD Reimer & KF499636 & KF499742 & NA & KF499826 & P. mutuki \\
\hline 1621 & Yeliu & Keelung & 06-Sep-09 & 1 & JD Reimer & KF499635 & KF499741 & KF499765 & KF499786 & P. mutuki \\
\hline 1622 & Yeliu & Keelung & 06-Sep-09 & 2 & JD Reimer & KF499646 & KF499725 & NA & KF499825 & $\begin{array}{l}\text { Zoanthus } \\
\text { gigantus }\end{array}$ \\
\hline 1623 & Yeliu & Keelung & 06-Sep-09 & 2 & JD Reimer & KF499634 & KF499740 & KF499766 & NA & P. mutuki \\
\hline 1624 & Yeliu & Keelung & 06-Sep-09 & 2 & JD Reimer & NA & NA & NA & NA & P. mutuki \\
\hline 1625 & Yeliu & Keelung & 06-Sep-09 & 2 & JD Reimer & KF499648 & KF499726 & NA & KF499824 & Z. gigantus \\
\hline 1626 & Yeliu & Keelung & 06-Sep-09 & 2 & JD Reimer & NA & NA & NA & NA & Z. gigantus \\
\hline 1627 & Yeliu & Keelung & 06-Sep-09 & 2 & JD Reimer & KF499678 & KF499719 & NA & KF499823 & Z. vietnamensis \\
\hline 1628 & Yeliu & Keelung & 06-Sep-09 & 2 & JD Reimer & KF499647 & KF499724 & NA & NA & Z. gigantus \\
\hline 1629 & Bitouchiao & Keelung & 06-Sep-09 & 2 & JD Reimer & KF499633 & KF499739 & NA & NA & P. mutuki \\
\hline
\end{tabular}


Table 1 Identity of zoanthid specimens from Taiwan examined in this study, their collection information, and associated GenBank accession numbers (Continued)

\begin{tabular}{|c|c|c|c|c|c|c|c|c|c|c|}
\hline 1630 & Bitouchiao & Keelung & 06-Sep-09 & 2 & JD Reimer & KF499632 & KF499738 & KF499768 & KF499822 & P. mutuki \\
\hline 1631 & Bitouchiao & Keelung & 06-Sep-09 & 2 & JD Reimer & KF499660 & KF499737 & KF499772 & KF499785 & P. tuberculosa \\
\hline 1632 & Bitouchiao & Keelung & 06-Sep-09 & 2 & JD Reimer & KF499650 & KF499723 & NA & KF499821 & Z. sansibaricus \\
\hline 1633 & Bitouchiao & Keelung & 06-Sep-09 & 4 & JD Reimer & KF499644 & KF499718 & NA & KF499784 & Z. gigantus \\
\hline 1634 & Bitouchiao & Keelung & 06-Sep-09 & 8 & JD Reimer & KF499659 & KF499736 & NA & NA & P. tuberculosa \\
\hline 1635 & Bitouchiao & Keelung & 06-Sep-09 & 8 & JD Reimer & KF499652 & KF499735 & KF499776 & KF499783 & $\begin{array}{l}\text { Palythoa sp. } \\
\text { "sakurajimensis" }\end{array}$ \\
\hline 1636 & Bitouchiao & Keelung & 06-Sep-09 & 2 & JD Reimer & KF499643 & KF499717 & NA & NA & Z. gigantus \\
\hline 1637 & Bitouchiao & Keelung & 06-Sep-09 & 2 & JD Reimer & KF499677 & KF499716 & NA & KF499789 & Z. vietnamensis \\
\hline 1638 & Bitouchiao & Keelung & 06-Sep-09 & 2 & JD Reimer & KF499676 & KF499715 & NA & KF499790 & Z. vietnamensis \\
\hline 1639 & Bitouchiao & Keelung & 06-Sep-09 & 2 & JD Reimer & KF499675 & KF499714 & NA & KF499791 & Z. vietnamensis \\
\hline 1640 & Bitouchiao & Keelung & 06-Sep-09 & 3 & JD Reimer & KF499658 & KF499734 & KF499774 & KF499782 & P. tuberculosa \\
\hline 1641 & Bitouchiao & Keelung & 06-Sep-09 & 3 & JD Reimer & KF499657 & KF499733 & KF499775 & KF499798 & P. tuberculosa \\
\hline 1642 & Yeliu & Keelung & 06-Sep-09 & Intertidal & JD Reimer & KF499639 & NA & KF499769 & KF499799 & P. mutuki \\
\hline 1654 & Zolo & Green I. & 06-Sep-10 & 1 & JD Reimer & KF499673 & KF499763 & NA & KF499801 & $\begin{array}{l}\text { Zoanthus } \\
\text { kuroshio }\end{array}$ \\
\hline 1655 & Zolo & Green I. & 06-Sep-10 & 1 & JD Reimer & KF499672 & KF499762 & NA & KF499788 & Z. kuroshio \\
\hline 1656 & Zolo & Green I. & 06-Sep-10 & 1 & JD Reimer & KF499656 & KF499761 & KF499773 & NA & P. tuberculosa \\
\hline 1657 & Zolo & Green I. & 06-Sep-10 & 1 & JD Reimer & KF499671 & KF499760 & NA & KF499793 & Z. kuroshio \\
\hline 1658 & Zolo & Green I. & 06-Sep-10 & 1 & JD Reimer & KF499631 & KF499759 & NA & KF499800 & P. mutuki \\
\hline 1659 & Zolo & Green I. & 06-Sep-10 & 1 & JD Reimer & KF499655 & KF499758 & NA & NA & P. tuberculosa \\
\hline 1660 & Zolo & Green I. & 06-Sep-10 & 1 & JD Reimer & KF499653 & KF499757 & NA & NA & P. tuberculosa \\
\hline 1661 & Zolo & Green I. & 06-Sep-10 & 1 & JD Reimer & KF499654 & KF499756 & NA & KF499803 & P. tuberculosa \\
\hline 1662 & Zolo & Green I. & 06-Sep-10 & 1 & JD Reimer & KF499674 & KF499755 & NA & KF499795 & Z. kuroshio \\
\hline 1663 & Zolo & Green I. & 06-Sep-10 & 1 & JD Reimer & KF499649 & KF499754 & NA & NA & Z. sansibaricus \\
\hline 1664 & Zolo & Green I. & 06-Sep-10 & 1 & JD Reimer & KF499642 & KF499753 & NA & NA & Z. gigantus \\
\hline 1665 & Zolo & Green I. & 06-Sep-10 & 1 & JD Reimer & NA & KF499752 & NA & NA & Z. gigantus \\
\hline 1666 & Zolo & Green I. & 06-Sep-10 & 1 & JD Reimer & NA & KF499751 & NA & NA & Z. gigantus \\
\hline 1667 & Zolo & Green I. & 06-Sep-10 & 1 & JD Reimer & KF499630 & KF499750 & NA & KF499808 & P. mutuki \\
\hline 1668 & Zolo & Green I. & 06-Sep-10 & 1 & JD Reimer & KF499679 & KF499749 & NA & NA & Z. vietnamensis \\
\hline 1669 & Zolo & Green I. & 06-Sep-10 & 1 & JD Reimer & KF499629 & KF499748 & NA & KF499804 & P. mutuki \\
\hline 1670 & Zolo & Green I. & 06-Sep-10 & 1 & JD Reimer & KF499628 & KF499747 & NA & KF499809 & P. mutuki \\
\hline TF1 & Shihlang & Green I. & 01-Nov-09 & 7 & T Fujii & NA & KF499731 & NA & NA & Parazoanthus sp. \\
\hline TF3 & Dabaisha & Green I. & 02-Nov-09 & 11 & TFujii & NA & NA & NA & NA & Palythoa sp. \\
\hline TF4 & Dabaisha & Green I. & 02-Nov-09 & 10 & T Fujii & NA & NA & NA & NA & $\begin{array}{l}\text { Acrozoanthus } \\
\text { australiae }\end{array}$ \\
\hline TF5 & Dabaisha & Green I. & 02-Nov-09 & 10 & T Fujii & NA & NA & NA & NA & A. australiae \\
\hline TF6 & Dabaisha & Green I. & 02-Nov-09 & 10 & TFujii & NA & NA & NA & NA & Palythoa sp. \\
\hline TF7 & Dabaisha & Green I. & 02-Nov-09 & 8 & T Fujii & NA & NA & NA & NA & Palythoa sp. \\
\hline TF8 & Dabaisha & Green I. & 02-Nov-09 & 4 & TFujii & KF499664 & KF499732 & GET & KF499813 & P. tuberculosa \\
\hline TF9 & Dabaisha & Green I. & 02-Nov-09 & 2 & TFujii & KF499645 & KF499713 & NA & NA & Z. gigantus \\
\hline TF10 & Dabaisha & Green I. & 02-Nov-09 & Intertidal & T Fujii & KF499651 & KF499712 & NA & $\mathrm{GET}(\mathrm{A})$ & Z. sansibaricus \\
\hline TF11 & Matichao & Green I. & 02-Nov-09 & 16 & T Fujii & NA & KF499728 & NA & NA & $\begin{array}{l}\text { Epizoanthus } \\
\text { illoricatus }\end{array}$ \\
\hline TF12 & Matichao & Green I. & 02-Nov-09 & 13 & T Fujii & KF499612 & KF499703 & KF499771 & KF499814 & $\begin{array}{l}\text { Palythoa } \\
\text { heliodiscus }\end{array}$ \\
\hline
\end{tabular}


Table 1 Identity of zoanthid specimens from Taiwan examined in this study, their collection information, and associated GenBank accession numbers (Continued)

\begin{tabular}{|c|c|c|c|c|c|c|c|c|c|c|}
\hline TF13 & Matichao & Green I. & 02-Nov-09 & 6 & T Fujii & NA & NA & NA & NA & Palythoa sp. \\
\hline TF14 & Matichao & Green I. & 02-Nov-09 & 3 & T Fujii & KF499670 & KF499727 & NA & KF499797 & Z. vietnamensis \\
\hline TF15 & Dabaisha & Green I. & 02-Nov-09 & 1 & T Fujii & KF499640 & KF499746 & NA & KF499815 & P. mutuki \\
\hline TF16 & Matichao & Green I. & 02-Nov-09 & 1 & E Hirose & KF499669 & KF499711 & NA & KF499802 & Z. vietnamensis \\
\hline TF17 & Gweiwan & Green I. & 04-Nov-09 & NA & T Fujii & KF499686 & KF499722 & NA & NA & $\begin{array}{l}\text { Hydrozoanthus } \\
\text { gracilis }\end{array}$ \\
\hline TF20 & Gweiwan & Green I. & 04-Nov-09 & 21 & T Fujii & NA & NA & KF499781 & NA & Palythoa sp. \\
\hline TF21 & Gweiwan & Green I. & 04-Nov-09 & 20 & T Fujii & NA & NA & NA & NA & Palythoa sp. \\
\hline TF22 & Gweiwan & Green I. & 04-Nov-09 & 8 & T Fujii & NA & NA & NA & NA & Palythoa sp. \\
\hline YI1 & Shihlang & Green I. & 01-Nov-09 & 2 & Y Irei & KF499681 & KF499708 & NA & KF499796 & Z. vietnamensis \\
\hline YI2 & Shihlang & Green I. & 01-Nov-09 & 5 & Y Irei & KF499614 & KF499702 & KF499779 & KF499810 & P. heliodiscus \\
\hline$Y \mid 3$ & Shihlang & Green I. & 01-Nov-09 & 8 & Y Irei & NA & NA & NA & NA & Z. gigantus \\
\hline YI4 & Shihlang & Green I. & 01-Nov-09 & 9 & Y Irei & KF499613 & KF499701 & KF499780 & NA & P. heliodiscus \\
\hline YI5 & Shihlang & Green I. & 01-Nov-09 & 9 & Y Irei & NA & KF499730 & NA & NA & Parazoanthus sp. \\
\hline YI6 & Shihlang & Green I. & 01-Nov-09 & 7 & Y Irei & NA & NA & NA & NA & P. tuberculosa \\
\hline YI7 & Shihlang & Green I. & 01-Nov-09 & 9 & Y Irei & NA & KF499729 & NA & NA & Parazoanthus sp. \\
\hline YI8 & Dabaisha & Green I. & 02-Nov-09 & 10 & Y Irei & KF499685 & KF499720 & KF499777 & KF499811 & $\begin{array}{l}\text { Palythoa sp. } \\
\text { "sakurajimensis" }\end{array}$ \\
\hline YI10 & Dabaisha & Green I. & 02-Nov-09 & 11 & Y Irei & NA & NA & NA & NA & H. gracilis \\
\hline YI11 & Dabaisha & Green I. & 02-Nov-09 & 11 & Y Irei & NA & NA & NA & NA & H. gracilis \\
\hline YI12 & Dabaisha & Green I. & 02-Nov-09 & 11 & Y Irei & NA & KF499721 & NA & NA & Parazoanthus sp. \\
\hline YI13 & Dabaisha & Green I. & 02-Nov-09 & 4 & Y Irei & NA & NA & NA & NA & P. tuberculosa \\
\hline YI14 & Dabaisha & Green I. & 02-Nov-09 & 4 & Y Irei & KF499641 & KF499700 & KF499770 & KF499812 & P. mutuki \\
\hline YI15 & Dabaisha & Green I. & 02-Nov-09 & 2 & Y Irei & NA & NA & NA & NA & Z. sansibaricus \\
\hline YI16 & Matichao & Green I. & 02-Nov-09 & 15 & Y Irei & NA & NA & NA & NA & E. illoricatus \\
\hline YI17 & Matichao & Green I. & 02-Nov-09 & 5 & Y Irei & NA & NA & NA & NA & Palythoa sp. \\
\hline YI18 & Matichao & Green I. & 02-Nov-09 & 5 & Y Irei & NA & NA & NA & NA & Palythoa sp. \\
\hline YI19 & Gweiwan & Green I. & 04-Nov-09 & 9 & Y Irei & KF499682 & KF499710 & NA & NA & A. australiae \\
\hline YI20 & Gweiwan & Green I. & 04-Nov-09 & 9 & Y Irei & KF499684 & KF499709 & NA & GET (D) & A. australiae \\
\hline YI21 & Sankuaiyan & Green I. & 04-Nov-09 & 20 & Y Irei & NA & NA & NA & NA & Palythoa sp. \\
\hline $\mathrm{YI} 22$ & Dabaisha & Green I. & 02-Nov-09 & Intertidal & Y Irei & NA & NA & NA & NA & P. mutuki \\
\hline $\mathrm{YI} 23$ & Dabaisha & Green I. & 02-Nov-09 & Intertidal & Y Irei & NA & NA & NA & NA & Z. sansibaricus \\
\hline YI24 & Dabaisha & Green I. & 02-Nov-09 & Intertidal & Y Irei & KF499680 & KF499707 & NA & KF499792 & Z. vietnamensis \\
\hline WLT1 & Wanli Tung & Kenting & Sept 2012 & 3 to 5 & SY Yang & NA & NA & NA & KF499817 & P. tuberculosa \\
\hline WLT2 & Wanli Tung & Kenting & Sept 2012 & 3 to 5 & SY Yang & NA & NA & NA & KF499828 & P. tuberculosa \\
\hline WLT3 & Wanli Tung & Kenting & Sept 2012 & 3 to 5 & SY Yang & NA & NA & NA & KF499829 & P. tuberculosa \\
\hline WLT4 & Wanli Tung & Kenting & Sept 2012 & 3 to 5 & SY Yang & NA & NA & NA & KF499830 & P. tuberculosa \\
\hline WLT5 & Wanli Tung & Kenting & Sept 2012 & 3 to 5 & SY Yang & NA & NA & NA & KF499831 & P. tuberculosa \\
\hline WLT6 & Wanli Tung & Kenting & Sept 2012 & 3 to 5 & SY Yang & NA & NA & NA & KF499832 & P. tuberculosa \\
\hline WLT7 & Wanli Tung & Kenting & Sept 2012 & 3 to 5 & SY Yang & NA & NA & NA & KF499833 & P. tuberculosa \\
\hline WLT8 & Wanli Tung & Kenting & Sept 2012 & 3 to 5 & SY Yang & NA & NA & NA & KF499834 & P. tuberculosa \\
\hline WLT9 & Wanli Tung & Kenting & Sept 2012 & 3 to 5 & SY Yang & NA & NA & NA & KF499835 & P. tuberculosa \\
\hline WLT10 & Wanli Tung & Kenting & Sept 2012 & 3 to 5 & SY Yang & NA & NA & NA & KF499836 & P. tuberculosa \\
\hline WLT11 & Wanli Tung & Kenting & Sept 2012 & 3 to 5 & SY Yang & NA & NA & NA & KF499837 & P. tuberculosa \\
\hline WLT12 & Wanli Tung & Kenting & Sept 2012 & 3 to 5 & SY Yang & NA & NA & NA & KF499838 & P. tuberculosa \\
\hline
\end{tabular}


Table 1 Identity of zoanthid specimens from Taiwan examined in this study, their collection information, and associated GenBank accession numbers (Continued)

\begin{tabular}{|c|c|c|c|c|c|c|c|c|c|c|}
\hline WLT13 & Wanli Tung & Kenting & Sept 2012 & 3 to 5 & SY Yang & NA & NA & NA & KF499839 & P. tuberculosa \\
\hline WLT14 & Wanli Tung & Kenting & Sept 2012 & 3 to 5 & SY Yang & NA & NA & NA & KF499840 & P. tuberculosa \\
\hline WLT15 & Wanli Tung & Kenting & Sept 2012 & 3 to 5 & SY Yang & NA & NA & NA & KF499841 & P. tuberculosa \\
\hline TWLK1 & Longkeng & Kenting & Dec 2011 & 3 to 5 & CY Kuo & NA & NA & NA & KF499818 & P. tuberculosa \\
\hline TWLK2 & Longkeng & Kenting & Dec 2011 & 3 to 5 & CY Kuo & NA & NA & NA & KF499842 & P. tuberculosa \\
\hline TWLK3 & Longkeng & Kenting & Dec 2011 & 3 to 5 & CY Kuo & NA & NA & NA & KF499843 & P. tuberculosa \\
\hline TWLK4 & Longkeng & Kenting & Dec 2011 & 3 to 5 & CY Kuo & NA & NA & NA & KF499844 & P. tuberculosa \\
\hline TWLK5 & Longkeng & Kenting & Dec 2011 & 3 to 5 & CY Kuo & NA & NA & NA & KF499845 & P. tuberculosa \\
\hline TWLK6 & Longkeng & Kenting & Dec 2011 & 3 to 5 & CY Kuo & NA & NA & NA & KF499846 & P. tuberculosa \\
\hline TWLK7 & Longkeng & Kenting & Dec 2011 & 3 to 5 & CY Kuo & NA & NA & NA & KF499847 & P. tuberculosa \\
\hline TWLK8 & Longkeng & Kenting & Dec 2011 & 3 to 5 & CY Kuo & NA & NA & NA & KF499848 & P. tuberculosa \\
\hline TWLK10 & Longkeng & Kenting & Dec 2011 & 3 to 5 & CY Kuo & NA & NA & NA & KF499850 & P. tuberculosa \\
\hline TWLK11 & Longkeng & Kenting & Dec 2011 & 3 to 5 & CY Kuo & NA & NA & NA & KF499851 & P. tuberculosa \\
\hline GIGW1 & Gweiwan & Green I. & Sept 2012 & NA & Y Nozawa & NA & NA & NA & KF499820 & P. tuberculosa \\
\hline GIGW2 & Gweiwan & Green I. & Sept 2012 & NA & Y Nozawa & NA & NA & NA & KF499859 & P. tuberculosa \\
\hline GIGW3 & Gweiwan & Green I. & Sept 2012 & NA & Y Nozawa & NA & NA & NA & KF499860 & P. tuberculosa \\
\hline GIGW4 & Gweiwan & Green I. & Sept 2012 & NA & Y Nozawa & NA & NA & NA & KF499861 & P. tuberculosa \\
\hline GIGW5 & Gweiwan & Green I. & Sept 2012 & NA & Y Nozawa & NA & NA & NA & KF499862 & P. tuberculosa \\
\hline PHWA1 & Wangan & Penghu Is. & Sept 2012 & 3 to 5 & $\begin{array}{l}\text { HJ Hsieh, } \\
\text { AC Chung }\end{array}$ & NA & NA & NA & KF499819 & P. tuberculosa \\
\hline PHWA2 & Wangan & Penghu Is. & Sept 2012 & 3 to 5 & $\begin{array}{l}\text { HJ Hsieh, } \\
\text { AC Chung }\end{array}$ & NA & NA & NA & KF499856 & P. tuberculosa \\
\hline PHWA4 & Wangan & Penghu Is. & Sept 2012 & 3 to 5 & $\begin{array}{l}\text { HJ Hsieh, } \\
\text { AC Chung }\end{array}$ & NA & NA & NA & KF499858 & P. tuberculosa \\
\hline PHWA9g1 & Wangan & Penghu Is. & Sept 2012 & 3 to 5 & $\begin{array}{l}\text { HJ Hsieh, } \\
\text { AC Chung }\end{array}$ & NA & NA & NA & KF499852 & P. tuberculosa \\
\hline PHWA9g2 & Wangan & Penghu Is. & Sept 2012 & 3 to 5 & $\begin{array}{l}\text { HJ Hsieh, } \\
\text { AC Chung }\end{array}$ & NA & NA & NA & KF499853 & P. tuberculosa \\
\hline PHWA9g3 & Wangan & Penghu Is. & Sept 2012 & 3 to 5 & $\begin{array}{l}\text { HJ Hsieh, } \\
\text { AC Chung }\end{array}$ & NA & NA & $\mathrm{NA}$ & KF499854 & P. tuberculosa \\
\hline PHWA9g4 & Wangan & Penghu Is. & Sept 2012 & 3 to 5 & $\begin{array}{l}\text { HJ Hsieh, } \\
\text { AC Chung }\end{array}$ & NA & NA & NA & KF499855 & P. tuberculosa \\
\hline
\end{tabular}

*All specimens in 2011 and 2012 newly reported in this study, other specimens collected as previously reported in Reimer et al. (2011b). Specimens with no sequence data identified only by external morphology. NA, not acquired.

DNA markers for zoanthid specimens were amplified in this study: (a) mitochondrial cytochrome oxidase subunit I (COI), (b) mitochondrial 16S ribosomal DNA (mt 16S rDNA), and (c) nuclear internal transcribed spacer region of ribosomal DNA (ITS-rDNA), using primers and amplification conditions respectively reported in Reimer et al. (2007a), Sinniger et al. (2010), and Reimer et al. (2007c) and Swain (2009). The ITS-rDNA of Symbiodinium spp. was amplified in this study using previously reported primers (White et al. 1990, Rowan and Powers 1992, Hunter et al. 1997) and amplification conditions reported in Reimer et al. (2007b).
All amplified products were visualized by $1.0 \%$ or $1.5 \%$ agarose gel electrophoresis, and positive products were treated with shrimp alkaline phosphate (SAP; Takara, Shiga, Japan) and exonuclease I. Sequencing was performed by MacroGen Japan (Tokyo, Japan) and Fasmac (Tokyo, Japan).

\section{Phylogenetic analyses}

Novel sequences obtained in this study were deposited in GenBank (accession nos.: KF499612-KF499862). For the mt $16 \mathrm{~S}$ rDNA analyses, only specimens of brachycnemic sequences $(=$ families Zoanthidae and Sphenopidae) 
were included in the analyses, and sequences from the Macroncnemina (= families Epizoanthidae and Hydrozoanthidae) were excluded to improve the resolution of resulting phylogenetic trees. For mitochondrial $\mathrm{COI}$ and 16S-rDNA alignments, sequences of Macrocnemina were utilized as outgroups, while for ITS-rDNA, an alignment of only Palythoa spp., was generated, owing to the high levels of divergence between sequences in Zoanthus + Acrozoanthus and problems acquiring accurate sequences for these groups. For ITS-rDNA sequences from Zoanthus + Acrozoanthus specimens, analyses were done by direct sequence comparison with BLAST (National Library of Medicine, Bethesda, Maryland, USA).

The obtained novel Symbiodinium ITS-rDNA sequences (Table 1) were aligned with previously obtained clade C Symbiodinium sequences (see the resulting phylogenetic tree), with Symbiodinium subclade C15/ C91 and related sequences as outgroups.

The three zoanthid alignments (all zoanthid COI, all zoanthid mt 16S rDNA, and Palythoa ITS-rDNA) were constructed as described in Reimer et al. (2012), following previous alignments as guides. All alignments were inspected by eye, and ambiguous sites (ambiguous or double peaks, $<2$ sites/alignment) were removed prior to the analyses. Three zoanthid alignment datasets were generated: (1) an 'all-zoanthid' COI alignment with 497 sites of 135 sequences, (2) an 'all-zoanthid' 16S-rDNA alignment with 685 sites of 120 sequences, and (3) a Palythoa ITS-rDNA alignment with 772 sites of 55 sequences. A Symbiodinium alignment of 275 sites of 101 sequences was generated. All four alignments are available from the corresponding author upon request.

For the phylogenetic analyses, the same methods were independently applied. Alignments were analyzed using neighbor-joining (NJ) and maximum-likelihood (ML) tests. ML was performed using PhyML (Guindon et al. 2010) with an input tree generated by BIONJ under the general time-reversible model (Lanave et al. 1984) of nucleotide substitution incorporating a discrete gamma distribution (eight categories) (GTR+). The discrete gamma distribution and base frequencies of the model were estimated from the dataset. PhyML bootstrap trees $(1,000$ replicates) were made utilizing the same parameters as the individual ML tree. Distances were calculated using a Kimura's two-parameter model (Kimura 1980). Support for the NJ branches was tested by bootstrap analysis (Felsenstein 1985) of 1,000 replicates. CLC Free Workbench 3.2.2 (Aarhus, Denmark) was used for the NJ phylogenetic analyses (1,000 replicates).

\section{Results}

COI

New COI sequences were obtained for 77 specimens. In the resulting ML tree from the COI alignment, Palythoa and Zoanthus + Acrozoanthus each formed large clades with generally high bootstrap values $(\mathrm{ML}=58 \%, \mathrm{NJ}=$ $96 \%$, and $\mathrm{ML}=96 \%, \mathrm{NJ}=99 \%$, respectively) (Figure 1 ). Macrocnemina sequences for the families Hydrozoanthidae and Parazoanthidae were basal to Brachycnemina.

Within the Palythoa clade, the species $P$. heliodiscus (sequences matching 100\% with previously reported GenBank accession sequence AB219214; $\mathrm{ML}=97 \%, \mathrm{NJ}=$ 99\%) and $P$. tuberculosa (sequences matching 100\% with previous P. tuberculosa sequences, e.g., AB128896 + others) ( $\mathrm{ML} \leq 50 \%, \mathrm{NJ}=\leq 50 \%)$ formed subclades. A single sequence (1623) of a $P$. mutuki specimen was included in the P. tuberculosa subclade. The majority of $P$. mutuki + unidentified Palythoa specimen (1595, 1597, 1635, and YI8) sequences (matching 100\% with AB128895 + others) formed a polytomy basal to $P$. heliodiscus. Very little (1-bp difference) or no variation in specimen sequences was seen within subclades. The sequence from specimen 1594 was derived from $P$. tuberculosa, and had a 1-bp difference from P. tuberculosa (e.g., AB128896 + others).

Within the Zoanthus + Acrozoanthus clade, four subclades were observed; one corresponding to $A$. australiae $(\mathrm{ML}=68 \%, \mathrm{NJ}=74 \%$; sequences matching $100 \%$ with HM171914), another to $Z$. gigantus ( $\mathrm{ML}=84 \%$, $\mathrm{NJ}=$ 93\%; sequences matching $100 \%$ with $\mathrm{AB} 128893+$ others), another to $Z$. sansibaricus ( $\mathrm{ML}=94 \%, \mathrm{NJ}=95 \%$; sequences matching $100 \%$ with $A B 194031+$ others), and another to $Z$. kuroshio and $Z$. vietnamensis $(M L=63 \%$, $\mathrm{NJ}=65 \%$; sequences matching $100 \%$ with $\mathrm{AB} 252668+$ others).

\section{mt 16S rDNA}

New mt 16S rDNA sequences were obtained for 73 specimens. In the resulting ML tree from the mt $16 \mathrm{~S}$ rDNA alignment, Palythoa and Zoanthus + Acrozoanthus each formed large clades $(\mathrm{ML} \leq 50 \%, \mathrm{NJ}=90 \%$, and $\mathrm{ML}=99 \%$, $\mathrm{NJ}=90 \%$, respectively) (Figure 2 ).

Within the Palythoa clade, species groupings were observed, although only $P$. heliodiscus (sequences matching $100 \%$ with previously reported DQ997844 + others) formed a well-supported single subclade (ML $=99 \%$, NJ = 99\%). Sequences from specimens YI8, 1595, 1597, and 1635 were identical to those of Palythoa sp. "sakurajimensis" sensu Reimer et al. (2007c) (DQ997863 and DQ997842). The sequence from 1594 was identical to sequence DQ997862 from another Palythoa sp. "sakurajimensis" specimen from Japan, and formed a subclade $(\mathrm{ML}=70 \%, \mathrm{NJ}=62 \%)$. Palythoa mutuki sequences formed a large subclade (matching 99\% to $100 \%$ with previously reported $P$. mutuki sequences, e.g., DQ997841 + others) (ML $\leq 50 \%, \mathrm{NJ}=63 \%)$ with a 1 -bp difference within the group, corresponding to $P$. mutuki 1 and $P$. mutuki 2 as previously reported (Reimer et al. 2006c, 2007c). Sequences from P. tuberculosa were 


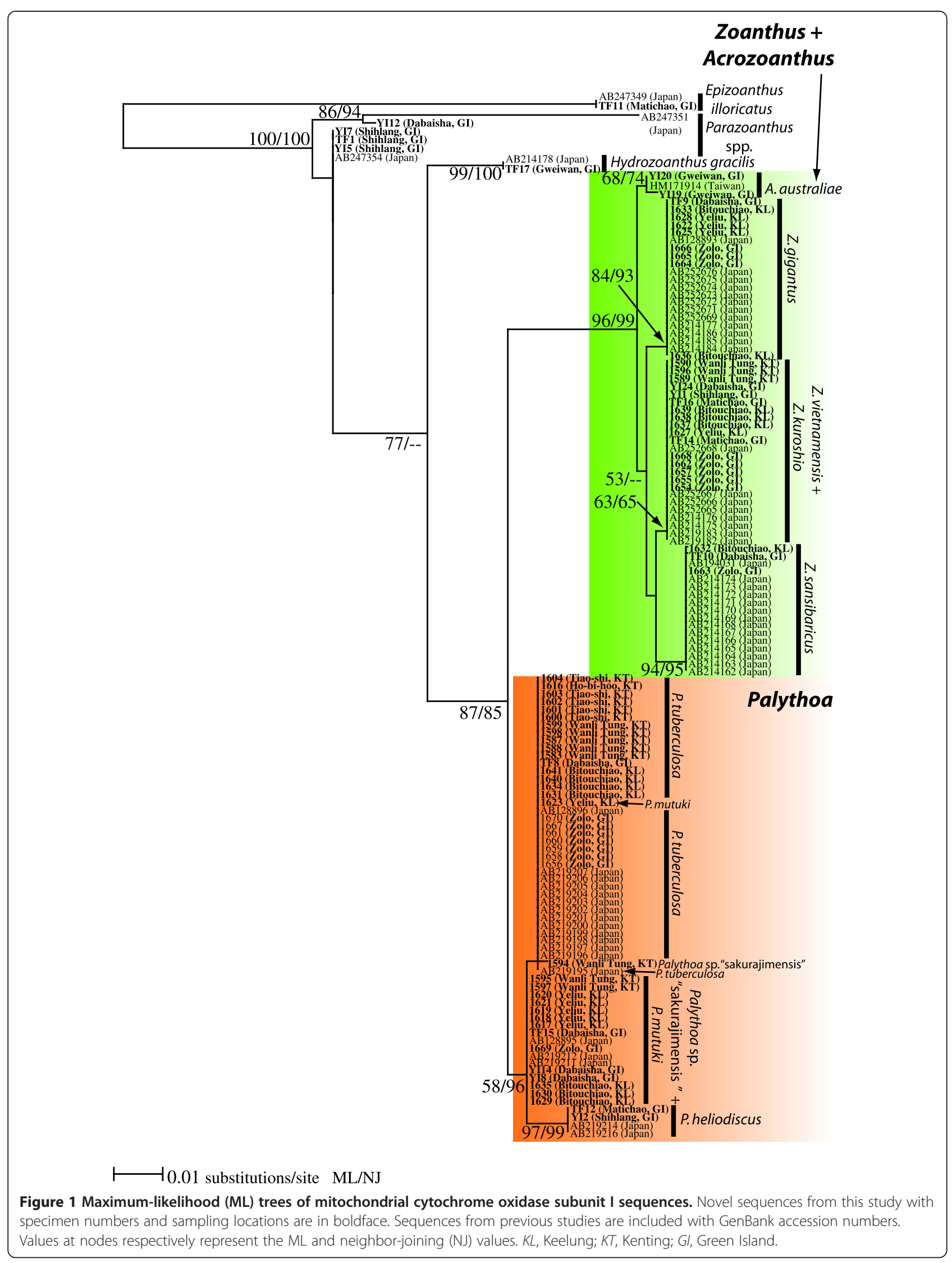


TF17 (Gweiwan, GI) Hydrozoanthus gracilis

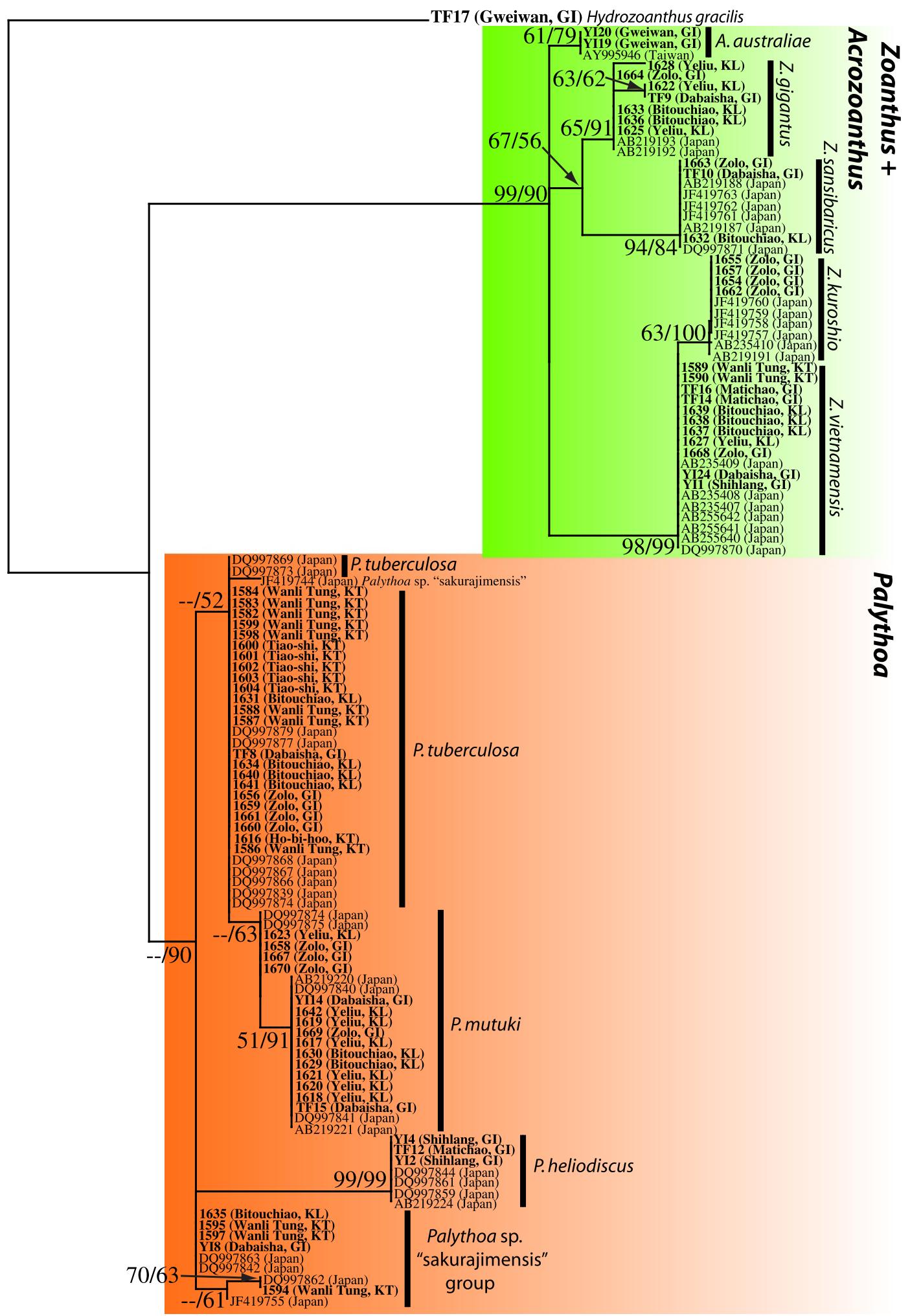

0.005 substitutions/site $\mathrm{ML} / \mathrm{NJ}$

Figure 2 Maximum-likelihood (ML) trees of mitochondrial 16S ribosomal DNA sequences. Novel sequences from this study with specimen numbers and sampling locations are in boldface. Sequences from previous studies are included with GenBank accession numbers. Values at nodes respectively represent $M L$ and neighbor-joining (NJ) values. KL, Keelung; KT, Kenting; Gl, Green Island. 
identical (sequences matching 99\% to $100 \%$ with DQ997879 + others), and formed a polytomy basal to P. mutuki. Very little (1-bp difference) or no variation in specimen sequences was seen within all subclades or groupings.

Within the Zoanthus + Acrozoanthus clade, four subclades were observed, one corresponding to $A$. australiae $(\mathrm{ML}=61 \%$, NJ $=79 \%$; sequences matching $100 \%$ with AY995946), another to $Z$. gigantus ( $\mathrm{ML}=$ $65 \%, \mathrm{NJ}=91 \%$; sequences matching 100\% with AB219193 + others), another to $Z$. sansibaricus ( $\mathrm{ML}=94 \%, \mathrm{NJ}=84 \%$; sequences matching 100\% with AB219188 + others), and another to $Z$. kuroshio and $Z$. vietnamensis ( $\mathrm{ML}=98 \%, \mathrm{NJ}=99 \%$; $Z$. vietnamensis matching $100 \%$ with $\mathrm{AB} 235409$ + others). Within the $Z$. kuroshio $+Z$. vietnamensis subclade, all $Z$. kuroshio specimens formed a subclade $(\mathrm{ML}=63 \%, \mathrm{NJ}=$ $100 \%$ ) with a 1-bp difference from $Z$. vietnamensis, matching $100 \%$ with previously reported Z. kuroshio sequences (e.g., AB235410 + others). Little (1 bp) or no variation in specimen sequences was seen within all Zoanthus + Acrozoanthus subclades.

\section{Palythoa ITS-rDNA}

ITS-rDNA sequences from collected Palythoa specimens all fit into one of the four large clades (Figure 3). Sequences from several specimens closely matched previously reported P. heliodiscus sequences (e.g., DQ997881), and formed a well-supported clade $(\mathrm{ML}=100 \%, \mathrm{NJ}=$ $100 \%)$. Some small variation was seen in sequences within this clade, notably a 35-bp insertion in the ITS-1 region of specimen YI2.

Sequences from three specimens (YI8, 1597, and 1635) formed a moderately well-supported clade $(\mathrm{ML}=74 \%$, $\mathrm{NJ}=100 \%$ ) with previously reported sequences of Palythoa sp. "sakurajimensis" from Japan (DQ997887).

Sequences from P. mutuki and P. tuberculosa formed large, moderately supported clades $(\mathrm{ML}=83 \%, \mathrm{NJ}=$ $98 \%$ and $\mathrm{ML}=53 \%, \mathrm{NJ}=100 \%$ for NJ, respectively), and much variation in sequences was seen in both clades.

\section{Zoanthus + Acrozoanthus ITS-rDNA}

ITS-rDNA sequences were only obtainable for a few of all collected specimens in this group, with many other PCR products having double peaks, consistent with high amounts of variation previously reported in this group (Reimer et al. 2007d). For the BLAST results, sequences from specimens TF10, 1625, and 1636 matched 96\% to $99 \%$ with previously reported sequences of $Z$. gigantus (e.g., DQ442438), those from specimens TF9 and 1632 matched $84 \%$ to $99 \%$ with previously reported sequences of $Z$. sansibaricus (AB214215 and AB214156, respectively), and those from specimen 1627 matched 97\% with previously reported sequences of $Z$. vietnamensis (DQ442470). No usable sequences from $A$. australiae were obtained.

\section{Symbiodinium ITS-rDNA}

Within the generated ML tree, three major groupings were observed (Figure 4). The largest grouping consisted of subclade $\mathrm{C} 1$ and related derived sequences, and included ITS2 sequences from specimens 1586, 1595, $1599,1617,1619,1620,1621,1622,1625,1630,1631$, 1633, 1635, 1640, 1641, 1642, 1658, 1661, 1669, TF8, YI8, YI14, GIGW1 to 5, PWHA1 to 4 , TWLK 1 to 11 , WLT1 to 15 , and previously reported sequences from Palythoa and Isaurus species from the Pacific and Atlantic. Subclades c67, c69, c33, and c39 were also within this group, which had low bootstrap support ( $\mathrm{ML}=51 \%, \mathrm{NJ} \leq$ $50 \%$ ). Within this group, one distinct subgrouping was observed, made up of four ITS2 sequences from Penghu $P$. tuberculosa specimens (PWHA9g1 to g4). This subclade had very high bootstrap support ( $\mathrm{ML}=98 \%, \mathrm{NJ}=100 \%)$.

Another grouping within the ML tree corresponded to subclade C3, and was basal to subclade C1. Within this grouping were subclades C22, C31, and C49, previously reported sequences from $P$. tuberculosa from Ishigaki, Japan, and sequences from specimens 1598, 1627, 1654, 1667, 1670, TF12, TF15, TF16, and YI2. There was also one subclade within this grouping, formed by sequences from 1632 and previously reported sequences from $Z$. sansibaricus.

The third major grouping consisted of sequences identical or closely related to sequence AB207184 from Symbiodinium in Z. kuroshio (= C15/C91-related). Sequences from specimens 1594, 1655, 1657, 1662, TF14, and YI1 were identical to AB207184, while ITS2 sequences from 1639 and YI24 formed a poorly supported separate clade $(\mathrm{ML}=50 \%, \mathrm{NJ} \leq 50 \%)$. Two sequences from Z. vietnamensis in Keelung (1638 and 1639) formed another subclade with high support $(\mathrm{ML}=90 \%$, $\mathrm{NJ}=86 \%$ ).

\section{Discussion \\ Zoanthid species identities and comparisons with previous morphological identifications}

Results from the phylogenetic analyses of zoanthid specimens in this study generally confirm conclusions previously reached by Reimer et al. (2011b). Identifications in the previous study of specimens utilizing morphological features corresponded to phylogenetic species groupings observed in this study in almost all cases. In particular, results for specimens of $Z$. sansibaricus, $Z$. gigantus, $Z$. kuroshio, A. australiae (also reported in Reimer et al. 2011a), P. heliodiscus, P. tuberculosa, and P. mutuki matched the 2011 identifications. As previously reported (Reimer et al. 2006c, 2007c), P. mutuki may consist of more than one species, and evidence for this can be seen in the mt $16 \mathrm{~S}$ rDNA tree with two separate groupings in this species, and in the large variety within the species' ITS-rDNA clade. Palythoa tuberculosa ITS-rDNA sequences from 


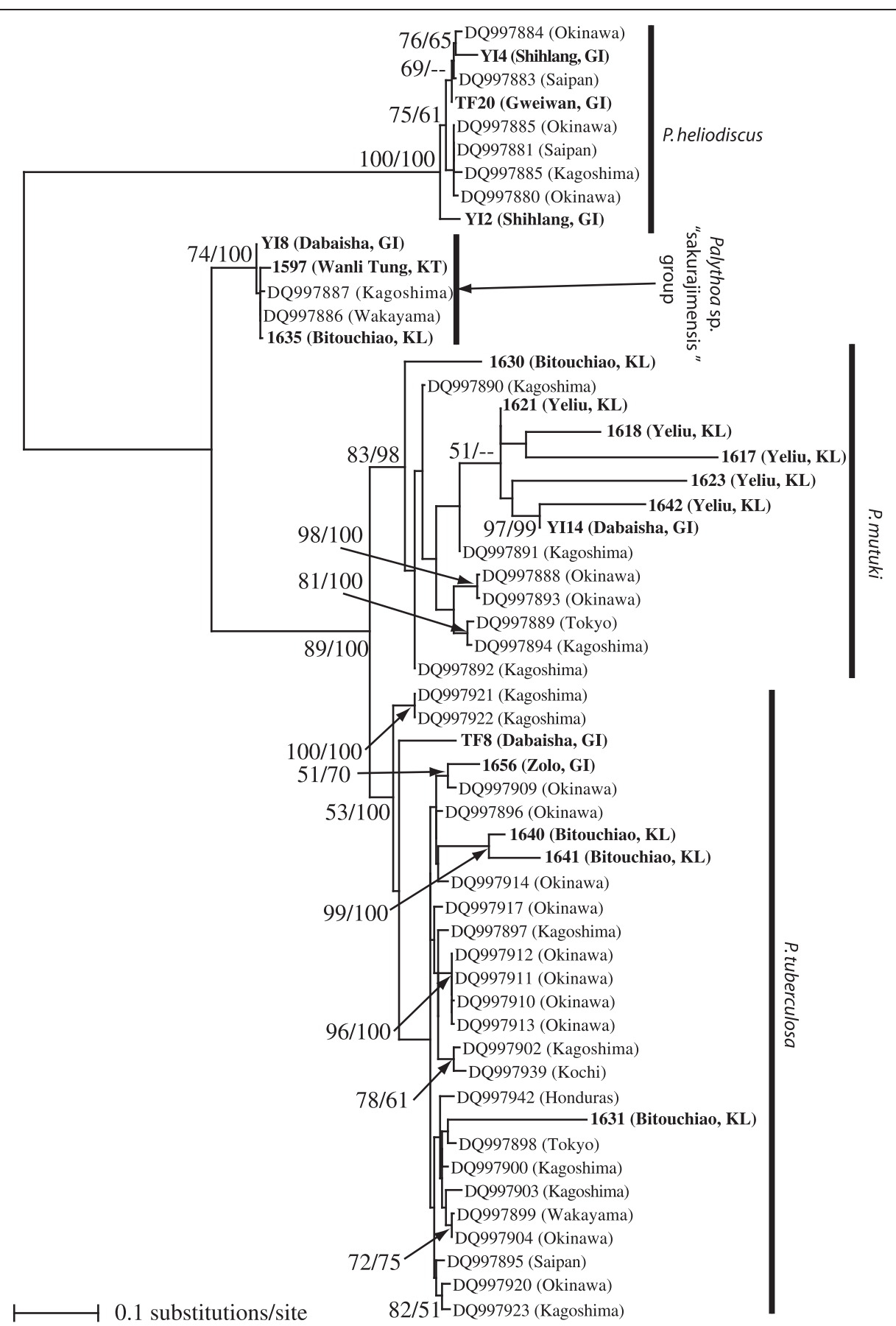

Figure 3 Maximum-likelihood (ML) trees of nuclear internal transcribed spacer region of ribosomal DNA (ITS-rDNA) sequences from Palythoas spp. Novel sequences from this study with specimen numbers and sampling locations are in boldface (species, specimen number). Sequences (GenBank accession number) from previous studies are in regular font. Values at nodes respectively represent ML and neighborjoining (NJ) values. KL, Keelung; KT, Kenting; Gl, Green Island.

Taiwan were often distinct from those of Japanese specimens but formed multiple clades. Given previous reports on P. tuberculosa (Reimer et al. 2007c) showing that this species has large amounts of ITS-rDNA variation, we feel that the explanation that this is one species is much more likely than the alternate hypothesis that there are many species in Taiwan (and Japan and the Indo-Pacific) with overlapping morphology and distributions.

One important finding from this study was the confirmation of subtle phylogenetic differences between $Z$. kuroshio and $Z$. vietnamensis (referred to as $Z$. aff. vietnamensis in Reimer et al. 2011b). It is known that 


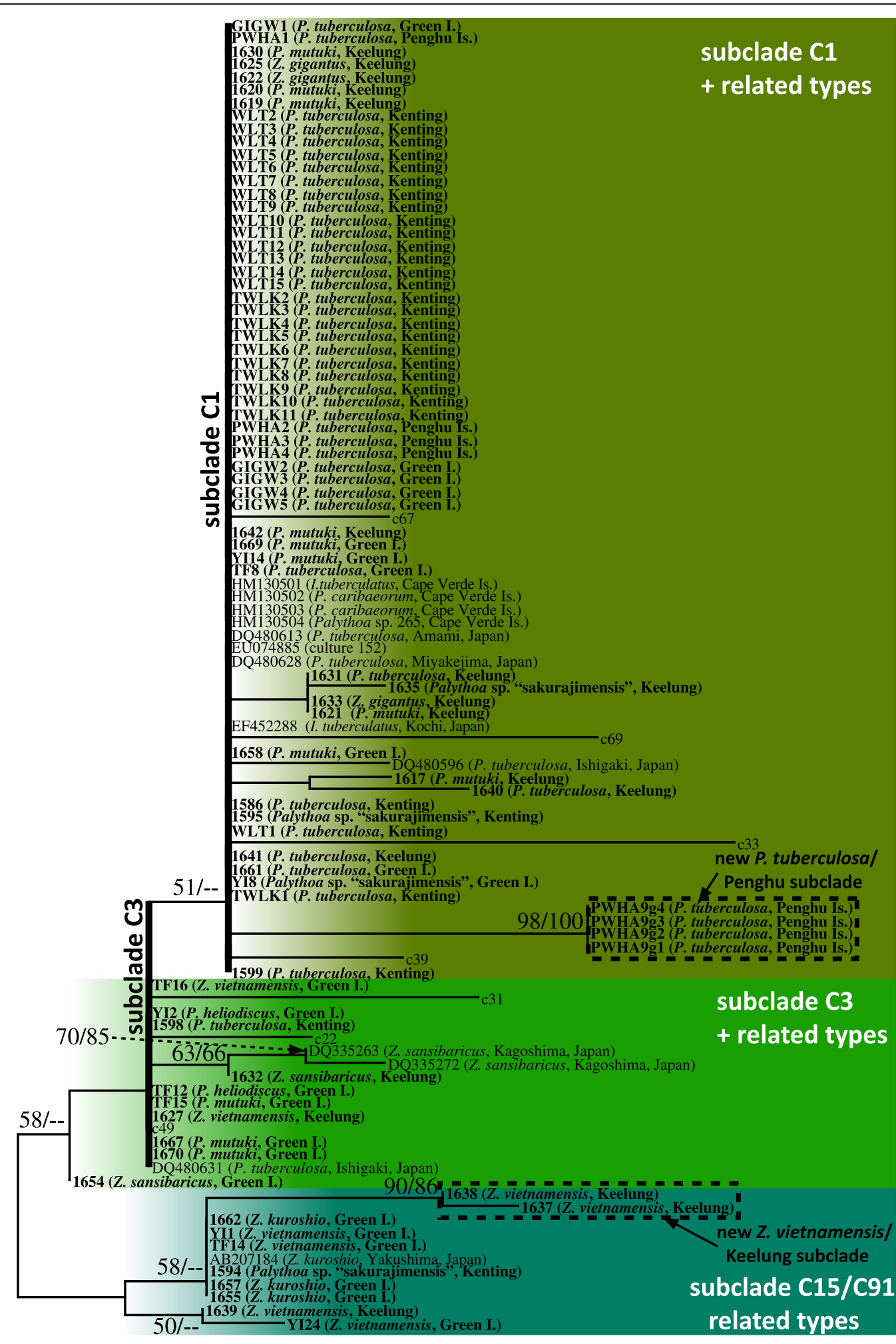

$\longmapsto 0.005$ substitutions/site ML/NJ

Figure 4 (See legend on next page.) 
(See figure on previous page.)

Figure 4 Maximum-likelihood (ML) tree of internal transcribed spacer 2 (ITS2) sequences of Symbiodinium from zoanthid specimens from Taiwan. Novel sequences from this study with specimen numbers (see Table 1), host species names, and sampling regions are in boldface. Subclades C1 and C3 (common Pacific types) are designated by thick vertical lines. Sequences from previous studies are included with GenBank accession numbers and/or subclade names (sensu LaJeunesse 2001). Potential new novel subclades are designated by dashed boxes. Values at nodes respectively represent $\mathrm{ML}$ and neighbor-joining (NJ) bootstrap values.

these two species are phylogenetically very closely related, and the status of $Z$. kuroshio as a valid independent species has been in question (Reimer et al. 2006b). However, results from this study confirm the separation of these two species. A small phylogenetic difference ( 1 to 2 bp in mt $16 \mathrm{~S}$ rDNA + COI sequences combined) was shown to be adequate to separate other zoanthid species (e.g., P. tuberculosa and P. mutuki) due to the slow rate of mtDNA mutations in Anthozoa (Shearer et al. 2002; Huang et al. 2008), and given the obvious morphological differences between these two groups (reminiscent of $P$. tuberculosa and $P$. mutuki), it is almost certain that these are two separate species. Further evidence comes the distribution of these two species in Taiwan, with Z. kuroshio only observed in coral reef environments (Kenting and Green Island) and $Z$. vietnamensis appearing most common at Keelung, a non-reef location. This is similar to the species' distribution in Japan, with $Z$. kuroshio most commonly seen in the southern Ryukyu Islands, and $Z$. vietnamensis reported from colder, more northerly locations and very rarely from the Ryukyus (Reimer 2010). Z. vietnamensis was described from Vietnam (Pax and Müller 1957) in the tropical South China Sea, but the coastline of Vietnam occasionally experiences winter sea surface temperatures of $<20^{\circ} \mathrm{C}$ (Japan Oceanographic Data Center; JODC), and morphologically, the Z. vietnamensis specimens reported here agree well with the original description, while Z. kuroshio was described from the Ryukyu Archipelago, which is influenced by the warm Kuroshio Current, and the type locality of Yakushima also has low winter sea surface temperatures of approximately $20^{\circ} \mathrm{C}$ (JODC). Zoanthus vietnamensis may be a subtropical/temperate species, and Z. kuroshio a tropical/subtropical species, with some overlap in their distributions. Another possibility is that these two species prefer different environments, with the "immersae" (= polyps embedded in well-developed coenenchyme; Pax 1910) Z. kuroshio on exposed reef crests and reef slopes (Irei et al. 2011) and the "liberae" (= polyps free and clear of coenenchyme) Z. vietnamensis in more sheltered locations. However, no investigation into the habitat preference of $Z$. vietnamensis has been conducted, and therefore, research into the sexual reproduction, ecology, and distribution of these two species should help further clarify the differences between these closely related species.
One additional finding of this study is that there is likely more than one undescribed Palythoa species in the northwestern Pacific. Some specimens in this study were seen in the phylogenies to be closely related to undescribed Palythoa sp. "sakurajimensis", with other specimens previously reported from southern Japan (Reimer et al. 2007c) and the Ogasawara Islands (Reimer et al. 2011a). These specimens were initially identified in Reimer et al. (2011b) as either P. mutuki or Palythoa sp. In this study and in previous studies, Palythoa sp. "sakurajimensis" specimen numbers were low compared to other Palythoa species, and colonies consisted only of one or a few polyps. Distinguishing this putative species from $P$. mutuki based on morphology is currently very difficult, with Palythoa sp. "sakurajimensis" being found at slightly deeper depths than P. mutuki. A concerted effort to collect more Palythoa sp. "sakurajimensis" specimens from each region and their examination are needed to formally describe this species.

Another potentially undescribed species is represented by specimen YI2, which was identified as $P$. heliodiscus based on COI and $\mathrm{mt} 16 \mathrm{~S}$ rDNA phylogenies, but it was previously identified as Palythoa sp. or Palythoa aff. heliodiscus based on morphology (Reimer et al. 2011b). A differing oral disc color (purple and/or green opposed to brown of $P$. heliodiscus) and ITS-rDNA results (Figure 3) indicate some differences between this specimen and $P$. heliodiscus. Similar insertions were seen in morphologically similar specimens from Singapore and Japan (P.A. Todd, T. Nishimura, and J.D. Reimer, unpublished data). As with Palythoa sp. "sakurajimensis", colonies of this putative species are likely to be rare, small, and cryptic.

For Isaurus tuberculatus reported in Reimer et al. (2011b), specimens were only observed by a third party and not collected, and no unforeseen results (e.g., undescribed species) are expected regarding their identity based on previous results (Muirhead and Ryland 1985; Reimer et al. 2008). Specimens of the "unknown zoanthid" from Reimer et al. (2011b) were also only observed but not collected, and these are very likely species within the recently described genus Microzoanthus Fujii \& Reimer 2011, based on their extremely small size and morphological similarity to that genus.

Results of this study demonstrate that mt $16 \mathrm{~S}$ rDNA sequences can more clearly define closely related species of zoanthids than can COI sequences. In this and previous 
studies, COI sequences failed to resolve the following species groups from each other: $Z$. vietnamensis $+Z$. kuroshio and P. mutuki + Palythoa sp. "sakurajimensis". A COI sequence from one specimen of $P$. mutuki was also seen within the $P$. tuberculosa clade. Future phylogenetic work on brachycnemic zoanthids should always include mt $16 \mathrm{~S}$ rDNA for this reason, and ITS-rDNA if possible.

\section{Symbiodinium spp. diversity in zoanthids in Taiwan}

Results of symbiont identification of Taiwanese zoanthids reflect other recent results reported from Japan, with some small but potentially important differences. Overall, association patterns as previously noted from different sites in Japan between various zoanthid host species and their symbionts were generally observed in this study. For example, the majority of Taiwanese $P$. tuberculosa (42 of 47 colonies or $89 \%$ ) and P. mutuki (9 of 12 colonies or $75 \%$ ) were associated with subclade $\mathrm{C} 1$ or closely related types, as reported in Japan (Reimer et al. 2006d). No Palythoa spp. were observed in association with clade D, which was reported from the Indian Ocean (Burnett 2002) and Singapore (Reimer and Todd 2009). Symbiodinium subclade $\mathrm{C} 1$ was shown to be a 'generalist' in terms of both associations and preferred environments, and together with subclade $\mathrm{C} 3$, is the most common IndoPacific type (LaJeunesse 2005). The lone A. australiae examined from Green Island was associated with clade D, as was previously reported from Kenting and Green Island (Reimer et al. 2010). Furthermore, Z. sansibaricus was associated with either C3-derived Symbiodinium (two of three colonies) or clade A (light tolerant) in shallow water, as was seen in Japan (Reimer et al. 2006e, 2007b). Finally, all three Z. kuroshio specimens examined had Symbiodinium ITS sequences identical to previously reported specimens from Japan (Reimer et al. 2006e) and Singapore (Reimer and Todd 2009).

However, some small differences from previous reports were seen. At Penghu, half (four of eight colonies) of P. tuberculosa specimens had a novel Symbiodinium C subclade derived from $\mathrm{C} 1$. This subclade had very high bootstrap support. These results suggest that $P$. tuberculosa can potentially associate with more than 1 clade C type. Palythoa tuberculosa is known to be a generalist species (Irei et al. 2011; Polak et al. 2011) with a high larval dispersal potential (Hirose et al. 2011; Polak et al. 2011), and associations with different symbiont types in different environments would seem to be a good strategy to adapt to different environments. However, while symbioses with clade D were observed in the Indian Ocean and Singapore, in the Pacific until now, $P$. tuberculosa is only known to associate with clade $\mathrm{C} 1 / \mathrm{C} 3$ or very closely related types, even in remote oceanic locations such as the Ogasawara (Reimer et al. 2011a) and Galapagos Islands (Reimer and Hickman 2009). The marine environment of Penghu is quite different from Kenting and other subtropical and tropical areas in southeastern Taiwan, as winter water temperatures may dip to $<15^{\circ} \mathrm{C}$ (Taiwan Central Weather Bureau), and often, the water is relatively turbid during the monsoon season (Huang et al. 2012a, b), and one or more of these factors may be contributed to the presence of this novel symbiont type. The occurrence of this divergent and novel type was somewhat unexpected, and further effort should be put into examining specimens from other areas near Penghu and the South China Sea, for which very few zoanthid data exist.

Another novel clade of symbionts was seen in $Z$. vietnamensis from Keelung. Additionally, it appeared that while Z. kuroshio from both Taiwan and Japan was associated only with one type of symbiont (C15/C91-derived, see Reimer et al. 2006e, 2007b), Z. vietnamensis is apparently more flexible in its association. Of the seven colonies examined in this study, two were associated with the new novel subclade (both at Keelung) and two with C15/C91 (identical to Z. kuroshio; both from Green Island), two were closely related to C15/C91 (Keelung/Green Island), and one was associated with subclade C3 (Keelung). Despite the examination of only seven colonies, the variety of types observed within $Z$. vietnamensis is surprising given its very close phylogenetic relationship with $Z$. kuroshio (Reimer et al. 2006b), and the fact that many other closely related zoanthid species are associated with similar or identical symbiont types, even in different oceans (e.g., Atlantic $P$. caribaeroum, sibling species to $P$. tuberculosa, harboring subclade $\mathrm{C} 1$ in Figure 1). Increasing specimen numbers with the inclusion of more specimens from the South China Sea (which is the type locality) and the Pacific coast of mainland Japan could also help clarify the nature of $Z$. vietnamensis' symbiont associations.

Finally, within this study, we examined zoanthid species for which no information on symbionts previously existed. Z. gigantus, P. heliodiscus, and Palythoa sp. "sakurajimensis" are not as common as the above mentioned zoanthid species (e.g., Irei et al. 2011) and are known to be distributed in both Taiwan and some locations in Japan (e.g., Reimer et al. 2011a). Specimen numbers of these species in this study are low $(n=2$ to 4$)$, and therefore, speculation on symbiont association patterns would be premature. The data acquired in this study should be compared to future, more in-depth studies. While all three of these species are usually associated with the "generalist" Symbiodinium C1 or C3 type, surprisingly, one Palythoa sp. "sakurajimensis" specimen from Kenting in southern Taiwan was associated with the C15/C91 type. This is the first time a Palythoa species has been reported to harbor this symbiont type. However, this species group contains multiple genotypes, and it is possible that it encompasses more than one species. Thus, any conclusions 
on host-symbiont associations for this group should be treated with caution.

\section{Conclusions}

While preliminary morphological results from Reimer et al. (2011b) were accurate in identifying known zoanthid species, it appears that there are still undescribed Palythoa species present in the northwestern Pacific. These species may be widely distributed yet low in numbers, often cryptic in their habitat, and with a small colony size. Phylogenetic and molecular analyses would be extremely useful for finding such unknown groupings. Likewise, methods and DNA markers utilized in this study could be used to confirm if free-living Sphenopus (family Sphenopidae) zoanthids in Taiwan are two species or not as previously theorized (Soong et al. 1999).

Despite the large specimen numbers in this study, it is clear that much work remains to understand zoanthidsymbiont associations in Taiwan. Recently, Symbiodinium spp. examinations using more rapidly evolving markers such as the non-coding region of the chloroplast-encoded $p s b A$ minicircle demonstrated differences even for symbionts with identical ITS2 sequences (e.g., LaJeunesse and Thornhill 2011). Acquisition of additional sequence data and eventually the formal description of zoanthidassociated symbiont species following molecular methods outlined in LaJeunesse et al. (2012) will allow for more exact characterizations of symbionts of zoanthids. However, from the results of this and other recent studies, it is clear that different zoanthid species have differing patterns of association with Symbiodinium.

This study, combined with Reimer et al. (2011b), is intended to serve as a basis for the molecular phylogenetic study of shallow-water zoanthids and their symbionts in Taiwan.

\section{Competing interests}

The authors declare that they have no competing interests.

\section{Authors' contributions}

JDR conceived the experiments, collected specimens, analyzed data, and wrote the paper. Yl, TF, and SYY collected specimens, and carried out the molecular genetic studies. All authors read and approved the final manuscript.

\section{Acknowledgements \\ The authors thank Drs. Yoko Nozawa and Chaolun Allen Chen (Biodiversity Research (enter, Academia Sinica; BRCAS) and members of their laboratories for their invaluable assistance in the field. Prof. Euichi Hirose (Univ. of the Ryukyus), Dr. Hernyi Justin Hsieh (Penghu Marine Biology Research Center, Fisheries Research Institute), Dr. Shashank Keshavmurthy, CY Kuo, HJ Hseih, and AC Chung (all BRCAS) also greatly assisted with the sample collection. The first author was funded by a grant-in-aid from the Japan Society for the Promotion of Science ('Wakate B' \#217700896), and the Rising Star Program and International Research Hub Project for Climate Change and Coral Reef/ Island Dynamics, both at the Univ. of the Ryukyus. Two anonymous reviewers' comments greatly improved the manuscript.}

Received: 19 May 2013 Accepted: 1 July 2013

Published: 17 October 2013

\section{References}

Burnett WJ (2002) Longitudinal variation in algal symbionts (zooxanthellae) from the Indian Ocean zoanthid Palythoa caesia. Mar Ecol Progr Ser 234:105-109

Burnett WJ, Benzie JAH, Beardmore JA, Ryland JS (1994) High genetic variability and patchiness in a common Great Barrier Reef zoanthid (Palythoa caesia). Mar Biol 121:153-160

Burnett WJ, Benzie JAH, Beardmore JA, Ryland JS (1997) Zoanthids (Anthozoa, Hexacorallia) from the Great Barrier Reef and Torres Strait, Australia: systematics, evolution and a key to species. Coral Reefs 16:55-68

Chen AC (1999) Analysis of scleractinian distribution in Taiwan indicating a pattern congruent with sea surface temperatures and currents: examples from Acropora and Faviidae corals. Zool Stud 38:119-129

Chen AC, Shashank K (2009) Taiwan as a connective stepping-stone in the Kuroshio Triangle and the conservation of coral ecosystems under the impacts of climate change. Kuroshio Sci 3:15-22

Felsenstein J (1985) Confidence limits on phylogenies: an approach using the bootstrap. Evolution 39:783-791

Guindon S, Dufayard JF, Lefort V, Anisimova M, Hordijk W, Gascuel O (2010) New algorithms and methods to estimate maximum-likelihood phylogenies: assessing the performance of PhyML 3.0. Syst Biol 59:307-321

Hirose M, Obuchi M, Hirose E, Reimer JD (2011) Timing of spawning and early development of Palythoa tuberculosa (Anthozoa, Zoantharia, Sphenopidae) in Okinawa. Japan Biol Bull 220:23-31

Huang D, Meier R, Todd PA, Chou LM (2008) Slow mitochondrial COI sequence evolution at the base of the metazoan tree and its implications for DNA barcoding. J Mol Evol 66:167-174

Huang YCA, Huang SC, Hsieh HJ, Meng PJ, Chen CA (2012a) Changes in sedimentation, sediment characteristics, and benthic macrofaunal assemblages around marine cage culture under seasonal monsoon scales in a shallow-water bay in Taiwan. J Exp Mar Biol Ecol 422-423:55-63

Huang YCA, Huang SC, Meng PJ, Hsieh HJ, Chen CA (2012b) Influence of strong monsoon winds on the water quality around a marine cage-cultured zone in a shallow and semi-enclosed bay in Taiwan. Mar Poll Bull 64:851-860

Hunter CL, Morden CW, Smith CM (1997) The utility of ITS sequences in assessing relationships among zooxanthellae and corals. Proc. 8th Int. Coral Reef Symp 2:1599-1602

Irei Y, Nozawa Y, Reimer JD (2011) Distribution patterns of five zoanthid species in Okinawa Island. Japan Zool Stud 50:426-433

Kamezaki M, Higa M, Hirose M, Suda S, Reimer JD (2013) Different zooxanthellae types in populations of the zoanthid Zoanthus sansibaricus along depth gradients in Okinawa. Japan Mar Biodivers 43:61-70

Karlson RH (1980) Alternative competitive strategies in a periodically disturbed habitat. Bull Mar Sci 30:894-900

Kimura M (1980) A simple method for estimating evolutionary rates of base substitutions through comparative studies of nucleotide sequences. J Mol Evol 16:111-120

LaJeunesse TC (2001) Investigating the biodiversity, ecology, and phylogeny of endosymbiotic dinoflagellates in the genus Symbiodinium using the ITS region: in search of a "species" level marker. J Phycol 37:866-880

LaJeunesse TC (2002) Diversity and community structure of symbiotic dinoflagellates from Caribbean coral reefs. Mar Biol 141:387-400

LaJeunesse TC (2005) "Species" radiations of symbiotic dinoflagellates in the Atlantic and Indo-Pacific since the Miocene-Pliocene transition. Mol Biol Evol 22:570-581

LaJeunesse TC, Thornhill DJ (2011) Improved resolution of reef-coral endosymbiont (Symbiodinium) species diversity, ecology, and evolution through psbA non-coding region genotyping. PLoS One 6:e29013

LaJeunesse TC, Loh WKW, Van Woesik R, Hoegh-Guldberg O, Schmidt GW, Fitt WK (2003) Low symbiont diversity in southern Great Barrier Reef corals, relative to those of the Caribbean. Limnol Oceanogr 48:2046-2054

LaJeunesse TC, Thornhill DJ, Cox EF, Stanton FG, Fitt WK, Schmidt GW (2004) High diversity and host specificity observed among symbiotic dinoflagellates in reef coral communities from Hawaii. Coral Reefs 23:596-603

LaJeunesse TC, Parkinson J, Reimer JD (2012) A genetics-based description of Symbiodinium minutum sp. nov. and S. psygmophilum sp. nov. (Dinophyceae), two dinoflagellates symbiotic with Cnidaria. J Phycol 48:1380-1391

Lanave C, Preparata G, Saccone C, Serio G (1984) A new method for calculating evolutionary substitution rates. J Mol Evol 20:86-93

Muirhead A, Ryland JS (1985) A review of the genus Isaurus Gray 1828 (Zoanthidea), including new records from Fiji. J Nat His 19:323-335 
Ong DCW, Reimer JD, Todd PA (2013) Morphologically plastic responses to shading in the zoanthids Zoanthus sansibaricus and Palythoa tuberculosa. Mar Biol 160:1053-1064

Pax F (1910) Studien an westindischen Actinien. Zool Jahrb Suppl 11:157-330

Pax F, Müller I (1957) Zoantharien aus Viet-Nam. Mem Mus Natl Hist Nat (Paris) 16:1-40

Pochon X, Gates RD (2010) A new Symbiodinium clade (Dinophyceae) from soritid foraminfera in Hawai'i. Mol Phylogenet Evol 56:492-497

Polak O, Loya Y, Brickner I, Kramarski-Winter E, Benayahu Y (2011) The widelydistributed Indo-Pacific zoanthid Palythoa tuberculosa: a sexually conservative strategist. Bull Mar Sci 87:605-621

Reimer JD (2010) Key to field identification of shallow water brachycnemic zoanthids (order Zoantharia: suborder Brachycnemina) present in Okinawa. Galaxea 12:23-29

Reimer JD, Hickman C (2009) Preliminary survey of zooxanthellate zoanthids (Cnidaria: Hexacorallia) of the Galápagos and associated symbiotic dinoflagellates (Symbiodinium spp.). Galápagos Res 66:14-19

Reimer JD, Todd PA (2009) Preliminary molecular examination of zooxanthellate zoanthid (Hexacorallia, Zoantharia) and associated zooxanthellae (Symbiodinium spp.) diversity in Singapore. Raffles Bull Zool Suppl 22:103-120

Reimer JD, Ono S, Fujiwara Y, Takishita K, Tsukahara J (2004) Reconsidering Zoanthus spp. diversity: molecular evidence of conspecificity within four previously presumed species. Zool Sci 21:517-525

Reimer JD, Ono S, Iwama A, Takishita K, Tsukahara J, Maruyama T (2006a) Morphological and molecular revision of Zoanthus (Anthozoa: Hexacorallia) from southwestern Japan, with descriptions of two new species. Zool Sci 23:261-275

Reimer JD, Ono S, Iwama A, Tsukahara J, Maruyama T (2006b) High levels of morphological variation despite close genetic relatedness between Zoanthus aff. vietnamensis and Zoanthus kuroshio (Anthozoa: Hexacorallia). Zool Sci 23:755-761

Reimer JD, Ono S, Takishita K, Tsukahara J, Maruyama T (2006c) Molecular evidence suggesting species in the zoanthid genera Palythoa and Protopalythoa (Anthozoa: Hexacorallia) are congeneric. Zool Sci 23:87-94

Reimer JD, Takishita K, Maruyama T (2006d) Molecular identification of symbiotic dinoflagellates (Symbiodinium spp.) from Palythoa spp. (Anthozoa: Hexacorallia) in Japan. Coral Reefs 25:521-527

Reimer JD, Takishita K, Ono S, Maruyama T, Tsukahara J (2006e) Latitudinal and intracolony ITS-rDNA sequence variation in the symbiotic dinoflagellate genus Symbiodinium (Dinophyceae) in Zoanthus sansibaricus (Anthozoa: Hexacorallia). Phycol Res 54:122-132

Reimer JD, Hirano S, Fujiwara Y, Sinniger F, Maruyama T (2007a) Morphological and molecular characterization of Abyssoanthus nankaiensis, a new family, new genus and new species of deep-sea zoanthid (Anthozoa: Hexacorallia: Zoantharia) from a northwest Pacific methane cold seep. Invert Systemat 21:255-262

Reimer JD, Ono S, Tsukahara J, Takishita K, Maruyama T (2007b) Non-seasonal clade-specificity and subclade microvariation in symbiotic dinoflagellates (Symbiodinium spp.) in Zoanthus sansibaricus (Anthozoa: Hexacorallia) at Kagoshima Bay. Japan Phycol Res 55:58-65

Reimer JD, Takishita K, Ono S, Maruyama T (2007c) Diversity and evolution in the zoanthid genus Palythoa (Cnidaria: Hexacorallia) utilizing nuclear ITS-rDNA Coral Reefs 26:399-410

Reimer JD, Takishita K, Ono S, Tsukahara J, Maruyama T (2007d) Molecular evidence suggesting intraspecific hybridization in Zoanthus (Anthozoa: Hexacorallia). Zool Sci 24:346-359

Reimer JD, Ono S, Tsukahara J, Iwase F (2008) Molecular characterization of the zoanthid genus Isaurus (Anthozoa: Hexacorallia) and its zooxanthellae (Symbiodinium spp). Mar Biol 153:351-363

Reimer JD, Ishikawa SA, Hirose M (2010) New records and molecular characterization of Acrozoanthus (Cnidaria: Anthozoa: Zoanthidae) from Taiwan. Mar Biodivers 41:313-323

Reimer JD, Hirose M, Yanagi K, Sinniger F (2011a) Marine invertebrate diversity in the oceanic Ogasawara Islands: a molecular examination of zoanthids (Anthozoa: Hexacorallia) and their Symbiodinium (Dinophycaea). Syst Biodivers 9:133-144

Reimer JD, Obuchi M, Irei Y, Fujii T, Nozawa Y (2011b) Shallow water brachycnemic zoanthids (Cnidaria: Hexacorallia) from Taiwan: a preliminary survey. Zool Stud 50:363-371

Reimer JD, Foord C, Irei Y (2012) Species diversity of shallow water zoanthids (Cnidaria: Anthozoa: Hexacorallia) in Florida. J Mar Biol, article ID 856079. doi:10.1155/2012/856079
Rowan R, Powers DA (1992) Ribosomal RNA sequences and the diversity of symbiotic dinoflagellates (zooxanthellae). Proc Natl Acad Sci USA 89:3639-3643

Ryland JS (1997) Budding in Acrozoanthus Saville-Kent, 1893 (Anthozoa: Zoanthidea). In: den Hartog JC (ed) Proceedings of the 6th International Conference on Coelenterate Biology,Noordwijkerhout, 1995. National Naturhistorisch Museum, Leiden, the Netherlands, pp 423-428

Shearer TL, van Oppen MJH, Romano SL, Wörheide G (2002) Slow mitochondrial DNA sequence evolution in the Anthozoa (Cnidaria). Mol Ecol 11:2475-2487

Sinniger F, Montoya-Burgess Jl, Chevaldonne P, Pawlowski J (2005) Phylogeny of the order Zoantharia (Anthozoa, Hexacorallia) based on mitochondrial ribosomal genes. Mar Biol 147:1121-1128

Sinniger F, Reimer JD, Pawlowski J (2010) The Parazoanthidae DNA taxonomy: description of two new genera. Mar Biodivers 40:57-70

Sinniger F, Ocaña OV, Baco AR (2013) Diversity of zoanthids (Anthozoa: Hexacorallia) on Hawaiian seamounts: description of the Hawaiian gold coral and additional zoanthids. PLoS One 8:e52607

Soong K, Shiau YS, Chen CP (1999) Morphological and life history divergence of the zoanthid, Sphenopus marsupialis off the Taiwanese coast. Zool Stud 38:333-343

Swain TD (2009) Phylogeny-based species delimitations and the evolution of host associations in symbiotic zoanthids (Anthozoa, Zoanthidea) of the wider Caribbean region. Zool J Linn Soc 156:223-238

Swain TD (2010) Evolutionary transitions in symbioses: dramatic reductions in bathymetric and geographic ranges of Zoanthidea coincide with loss of symbioses with invertebrates. Mol Ecol 19:2587-2598

Tchernov D, Gorbunov MY, de Vargas C, Yadav SN, Milligan AJ, Haggblom M, Falkowski PG (2004) Membrane lipids of symbiotic algae are diagnostic of sensitivity to thermal bleaching in corals. Proc Natl Acad Sci U S A 101:13531-13535

White TJ, Bruns T, Lee S, Taylor WJ (1990) Amplification and direct sequencing of fungal ribosomal genes for phylogenetics. In: Innis MA, Gelfanfd DH, Sninsky JJ, White TJ (eds) PCR protocols: a guide to methods and applications. Academic Press, San Diego, CA, pp 315-322

doi:10.1186/1810-522X-52-38

Cite this article as: Reimer et al: Molecular analyses of shallow-water zooxanthellate zoanthids (Cnidaria: Hexacorallia) from Taiwan and their Symbiodinium spp. Zoological Studies 2013 52:38.

\section{Submit your manuscript to a SpringerOpen ${ }^{\circ}$ journal and benefit from:}

- Convenient online submission

- Rigorous peer review

- Immediate publication on acceptance

- Open access: articles freely available online

- High visibility within the field

- Retaining the copyright to your article

Submit your next manuscript at $>$ springeropen.com 